\title{
Domain wall evolution at nanowires in terms of 3D LLG equation initial-boundary problem
}

\author{
S. Leble \\ Immanuel Kant Baltic Federal University, 236041 Kaliningrad, Russia \\ lebleu@mail.ru
}

PACS 75.70.Cn, 05.30.d, 02.20.a

DOI 10.17586/2220-8054-2021-12-1-42-59

\begin{abstract}
A theory of a domain wall creation and propagation is built on a linearized version of the transformed Landau-Lifshitz-Gilbert equation. The Lakshmanan-Nakamura stereo-graphic transform, after extra exponential transformation, and, next - linerization partially save information of the original nonlinearity that allows one to keep the domain wall dynamics, form and properties. For cylindrical-symmetric wire geometry, the conventional orthonormal Bessel basis, combined with projecting operators technique applied to subspaces of directed propagation of domain walls is constructed. The physically significant problems of the dynamics switching at points far and close from a wire ends are formulated and its solutions are presented in the frame of the Fourier method. Stationary solutions are found and the wall structure along the wire and propagation plots are drawn.
\end{abstract}

Keywords: Nanowire magnetization dynamics, domain wall creation, Landau-Lifshitz-Gilbert equation, Lakshmanan-Nakamura transform, initial-boundary problem.

Received: 11 January 2021

Revised: 25 January 2021

\section{Introduction}

The problem of domain wall (DW) creation and propagation was studied in many sources theoretically, e.g. in [1], and experimentally [1-5]. Historically, starting from the seminal Brown paper [6]: "striking success of micromagnetics has been its contribution to the theory of single-domain particles." The concept goes back to the 1930 paper of Frenkel-Dorfman [7], where they concluded from energy considerations that "if a particle is sufficiently small, it will not break up into domains but will be uniformly magnetized".

Brown equations [6] has a direct link to Landau-Lifshitz ones [8], see, e.g. [9]. Touching the DW creation and dynamics paradigm, that in its minimal 1D version relates the one-domain case, we have a simple stationary problem solution [10]. In more complicated cases the Landau-Lifshitz-Gilbert (LLG) equation can describe the dynamics of a DW in the magnetic core of circular cross-section at amorphous nanowires and glass-coated bistable microwires $[11,12]$. The DW propagation is characterized by high velocity propagation [13] and, therefore, there are several important applications [14,15]. Numerous applications are realized for magnetoelectric effects that are observed in special structures [16]. For a general exposition of the field, look [17]. The 1D-2D-3D LLG equations may be derived from Heisenberg chain equation by transition to continuous description and Gilbert term account [18]. The anisotropy term may also be added as phenomenological.

The Landau-Lifshitz equation, by Lakshmanan-Nakamura stereographic projection of the unit sphere of spin onto a complex plane [19], with extra exponential map [20] is transformed to nonlinear complex equation, convenient for further DW dynamics investigations [11]. In [19] it is shown that the effect of the Gilbert damping term is a mere rescaling of time by a complex constant. There are important results obtained for the complete LLG equations, including exact solutions, for example, ones, built by the Hirota method [21] and by a direct method [20]. These solutions, however do not satisfy the boundary conditions, that follow from general electrodynamics. Let us mention that a presence of stress field in amorphous medium influence the DW dynamics [24] so as defects influence implies a modification of the LLG equation to account the magnetic inhomogeneities that manifests in a DW acceleration [25].

We consider a DW creation as Barkhausen jump [9,22] example at nano- or microwire either far from end by pulse, or switching action of constant external magnetic field by the wires' end. These two problems are formulated on base of the transformed 3DLLG equation and, next, linearized the result. The isotropic matter version of the LLG equation parameters are chosen as independent on coordinates; for this case of physics, review [11]. Such a choice of the equations' approximation allows one to choose the correct boundary conditions in the context of the mentioned matter electrodynamics [23].

List the content by sections, in

- Sec. 2. We start with nonlinear LLG Lakshmanan-Nakamura-exponential transform, writing the resulting equation in cylindrical coordinates, convenient for circular cross-section wires geometry account. 
- Sec. 3. Derivation of boundary conditions at surfaces of the cylindrical wire.

- Sec. 4. We write the problem solution in terms of cylindrical functions series by Fourier method (division of variables). We also derive and study the dispersion relations and build projecting operators on dispersion branches subspaces, related to DW propagation direction.

- Sec. 5. Two physically significant problems of a DW creation are formulated. The first is related to DW generation by a local pulse, by a few-turn coil, far from ends and, the second, study a DW generation at ends by homogeneous field switching on. For the first case, we formulate and solve a Cauchy problem with initial $\delta$-distribution of magnetization.

- Sec. 6 is devoted to the stationary solution and magnetization vector presentation for a DW translation's mode. The result is illustrated by figures of the magnetization $z$-profiles.

\section{3DLLG for unit magnetization vector density}

\subsection{From Heisenberg chain equation to LLG}

Starting from Heisenberg chain equations, with anisotropy and Gilbert terms account, we go to continuous description [18,23], i.e. introduce the spin density field, re-scaled as $\vec{S} \rightarrow \vec{m}$, to unit magnetization vector field, having:

$$
-\frac{\partial \vec{m}}{\partial t}=J \vec{m} \times \nabla^{2} \vec{m}+K(\vec{m} \cdot \vec{n}) \vec{m} \times \vec{n}+\gamma \vec{m} \times \vec{H}-\alpha \vec{m} \times \frac{\partial \vec{m}}{\partial t},
$$

where $\gamma=\mu g \mu_{B}$ is gyromagnetic ratio, the product of the magnetic permeability $\mu$, Landé $g$-factor and Bohr magneton $\mu_{B} ; \alpha \ll 1$ is the damping Gilbert parameter, $J>0$ is the isotropic exchange energy coupling constant, $K$ is the strength of an effective easy-axis anisotropy, $\vec{H}=(0,0, H)$ - applied longitudinal magnetic field, $\vec{n}$ is the unit vector along $z$, see, e.g. [11]

\subsection{Transformations of 3DLLG (1)}

Following [11,19] it is useful to introduce a complex variable $\Omega(\vec{r}, t)$ such that:

$$
\exp (\Omega)=\frac{m^{x}+i m^{y}}{1+m^{z}}
$$

The structure of magnetization field then is defined mainly by the mentioned form of (2), while (1) transforms as:

$$
(i+\alpha) \Omega_{t}+J \nabla^{2} \Omega+\left(K-J(\nabla \Omega)^{2}\right) \tanh \left(\frac{\Omega+\Omega^{*}}{2}\right)=\gamma H .
$$

Such form yields a possibility to obtain interesting, physically reasonable particular solutions [11, 20]. Generally, the variable $\Omega$ is convenient to formulate an initial-boundary problem of a DW evolution, via an inversion of the transform (2). It opens the way to particular (in relation to LLG) linearization of the problem, that we study in this work.

Cylindrical variables for the Laplacian yields:

$$
\nabla^{2}=\Delta_{\perp}+\frac{\partial^{2}}{\partial z^{2}}
$$

where:

$$
\Delta_{\perp}=\Delta_{\rho}+\frac{1}{\rho^{2}} \frac{\partial^{2}}{\partial \varphi^{2}},
$$

in which:

$$
\Delta_{\rho}=\frac{1}{\rho} \frac{\partial}{\partial \rho} \rho \frac{\partial}{\partial \rho} .
$$

The linearization of the equation (3) gives:

$$
(\alpha+i) \Omega_{t}+J \nabla^{2} \Omega+K \Re \Omega=\gamma H .
$$

Let us conventionally split the general solution $\Omega^{l}=\Omega^{\prime}+\Omega^{i}$ : to the general one $\Omega^{\prime}$ for homogeneous equation:

$$
(\alpha+i) \Omega_{t}^{\prime}+J \nabla^{2} \Omega^{\prime}+K \Re \Omega^{\prime}=0,
$$

and a particular solution for the inhomogeneous one:

$$
(\alpha+i) \Omega_{t}^{i}+J \nabla^{2} \Omega^{i}+K \Re \Omega^{i}=\gamma H .
$$




\section{On statement of problem. Boundary conditions. Transverse modes}

\subsection{Real/Imaginary parts division}

3.1.1. Homogeneous equation and magnetization field. Let the solution of the homogeneous equation is presented as:

$$
\Omega^{\prime}=R+i S
$$

using the inverse to (2) relations we write:

$$
\begin{gathered}
\Re \Omega^{\prime}=R=\ln \left(\frac{1-m^{z}}{1+m^{z}}\right)=\ln \left(1-m^{z}\right)-\ln \left(1+m^{z}\right), \\
\exp \left[2 i \Im \Omega^{\prime}\right]=\exp [2 i S]=\frac{m^{x}+i m^{y}}{m^{x}-i m^{y}} .
\end{gathered}
$$

3.1.2. Boundary conditions for real and imaginary parts of $\Omega^{\prime}$. Let us take a body of a magnetic surrounded by dielectric. The magnetization vector $\vec{M}$ is introduced as:

$$
\vec{B}=\vec{H}+4 \pi \vec{M} \text {. }
$$

The continuity of the normal component of the vector $\vec{B}$ yields:

$$
H_{n i}+4 \pi M_{n i}=H_{n e}+4 \pi M_{n e},
$$

index " $i$ " marks internal, " $e$ " - external vectors with respect to an interface under consideration. Suppose the magnetization of the (external) medium, that is labeled by $e$, is zero, then:

$$
H_{n i}+4 \pi M_{n i}=H_{n e} .
$$

Similarly, from the continuity of the tangent component of the vector $\vec{H}$ we derive that:

$$
H_{t i}=B_{t i}-4 \pi M_{t i}=H_{t e}=B_{t e} .
$$

For an isotropic paramagnetic inside the body $\vec{B}_{i}=\mu_{i} \vec{H}_{i}$, while for the external one $\vec{B}=\vec{H}$, hence:

$$
\mu_{i} H_{t i}-4 \pi M_{t i}=H_{t e}=H_{t e},
$$

therefore:

For the normal component, similarly:

$$
\begin{gathered}
\frac{4 \pi}{\mu_{i}-1} M_{t i}=H_{t i}=H_{t e}, \\
M_{t i}=\frac{\mu_{i}-1}{4 \pi} H_{t e} .
\end{gathered}
$$

$$
\begin{gathered}
B_{n e}=B_{n i}=H_{n i}+4 \pi M_{n i}=\mu_{i} H_{n i}=H_{n e}, \\
M_{n i}=\left(\mu_{i}-1\right) H_{n i}=\frac{\mu_{i}-1}{4 \pi \mu_{i}} H_{n e} .
\end{gathered}
$$

The relations (21) and (20) pave the way to a boundary conditions for magnetic moment formulation.

A ferromagnetic boundary vicinity is characterized by "incomplete" number of nearest neighbours and, hence, lower exchange forces sum. It is hence have properties of a paramagnetic layer [23].

For the case of a wire lying along $z$-axis in a solenoid with the magnetic field $\vec{H} \| z$, co-axially, the normal component of $\vec{M}$ is zero. Hence:

$$
\left.m_{\rho}\right|_{\rho=\rho_{0}}=0 \text {. }
$$

The tangential components $m_{z}, m_{\phi}$ are proportional to external (solenoid) magnetic field. If the field is strictly parallel $z$,

If, however, on the basis of:

$$
\left.m_{\phi}\right|_{\rho=\rho_{0}}=0
$$

$$
M_{t i}=\frac{\mu_{i}-1}{4 \pi} H_{t e},
$$

taking $\mu_{i}-1 \approx 0$ into account, by (11), we suppose:

$$
\left.\Re \Omega^{\prime}(\varphi, \rho, z, t)\right|_{\rho=\rho_{0}}=R\left(\varphi, \rho_{0}, z, t\right)=0,
$$

where $\rho_{0}$ is the wire radius. Note, that nonzero condition may also be incorporated into the statement of problem. 


\section{Fourier method application}

\subsection{Division of variables}

The basic system for the $\mathrm{Re} / \mathrm{Im}$, or $R, S$ parts of the complex field $\Omega^{\prime}$ reads:

$$
\begin{gathered}
\alpha R_{t}+K R+J \nabla^{2} R=S_{t}, \\
R_{t}+\alpha S_{t}+J \nabla^{2} S=0 .
\end{gathered}
$$

It allows the division of variables, put $R=R_{d}+$ c.c., same for $S$,

$$
R_{d}(\varphi, \rho, z, t)=\exp [i \nu \varphi] \Sigma(\rho) Z(z, t), S_{d}=\exp [i \nu \varphi] \Sigma(\rho) W(z, t),
$$

which gives for (24):

$$
\Sigma Z_{t}+\alpha \Sigma W_{t}+J\left(W \Delta_{\rho} \Sigma-W \frac{\nu^{2}}{\rho^{2}} \Sigma+\frac{\partial^{2} W}{\partial z^{2}} \Sigma\right)=0
$$

Next step, after division by $\Sigma Z$ is:

$$
\frac{Z_{t}}{Z}+\alpha \frac{W_{t}}{W}+J\left(\frac{\Delta_{\rho} \Sigma}{\Sigma}-\frac{\nu^{2}}{\rho^{2}}+\frac{W_{z z}}{W}\right)=0 .
$$

The equation (23) transforms similarly.

\subsection{Spectral problem}

Conventionally, we put:

$$
\frac{\Delta_{\rho} \Sigma}{\Sigma}-\frac{\nu^{2}}{\rho^{2}}=-\lambda
$$

or, rewriting it as:

$$
\Delta_{\rho} \Sigma-\frac{\nu^{2}}{\rho^{2}} \Sigma=-\lambda \Sigma
$$

we arrive at the Bessel equation. We should add zero boundary conditions for $Z$ at $\rho_{0}$, see (22), having:

$$
\Sigma\left(\rho_{0}\right)=0 .
$$

Also, adding the finite-value condition at $\rho=0$, we have the Bessel basis.

Otherwise, the standard manipulations yields:

$$
\rho^{2} \frac{\partial^{2} \Sigma}{\partial \rho^{2}}+\rho \frac{\partial \Sigma}{\partial \rho}-\nu^{2} \Sigma+\lambda \rho^{2} \Sigma=0
$$

denoting: $\lambda \rho^{2}=r^{2}$, one goes to the Bessel equation for:

$$
J_{\nu}(r)=J_{\nu}(\sqrt{\lambda} \rho)
$$

If $\sqrt{\lambda}=\beta$

$$
\int_{0}^{\rho_{0}} J_{\nu}\left(\beta_{\nu, n} \rho\right) J_{\nu}\left(\beta_{\nu, m} \rho\right) d \rho=N_{\nu n} \delta_{n m}
$$

the spectrum $\beta_{\nu, m}\left(\rho_{0}\right)$, for each $\nu$ is determined by the equation:

$$
J_{\nu}\left(\beta \rho_{0}\right)=0, \quad \beta_{\nu, m}\left(\rho_{0}\right) \rho_{0}=\epsilon_{m}(\nu),
$$

therefore:

$$
\lambda_{\nu, m}=\beta_{\nu, m}^{2}=\frac{\epsilon_{m}^{2}(\nu)}{\rho_{0}^{2}}
$$

So, $\epsilon_{m}(\nu)$ are the zeros of the Bessel functions. In the case of $\nu=0, \epsilon_{1}(0) \approx 2.4$, hence $\lambda_{0,1}=\frac{(2.4)^{2}}{\rho_{0}^{2}}$. Taking $\rho_{0}=$ $100 \mathrm{~nm}=10^{-7} \mathrm{~m}$, we get $\lambda_{0,1}=\frac{(2.4)^{2}}{10^{-14}} \approx 5.8 \times 10^{14}$. A method of the zeros evaluation for $J_{\nu}$ is suggested [26], where the values are also listed. 


\subsection{Unit expansion}

Let us write the unit expansion, that is necessary for in-homogeneous (with nonzero rhs) equation (9) solution, using the Bessel function $J_{0}(r)$ as example. For the function $f(r), r \in[0,1]$, for $\nu=0$, the following expansion is valid:

$$
f(r)=\sum_{m=1}^{\infty} a_{m} J_{0}\left(\epsilon_{m}(0) r\right)
$$

with the coefficients, defined by:

$$
a_{n}=\frac{1}{N_{0 n}} \int_{0}^{1} f(r) J_{0}\left(\epsilon_{n}(0) r\right) r d r
$$

If $f(\rho)=1, \rho \leq \rho_{0}$, one has:

$$
a_{n}=\frac{1}{N_{0 n}} \int_{0}^{1} J_{0}\left(\epsilon_{n}(0) r\right) r d r
$$

By the textbook formula:

$$
\int_{0}^{a} J_{0}(r) r d r=a J_{1}(a)
$$

for $\epsilon_{n}(0)=\kappa$ :

$$
\int_{0}^{a} J_{0}(\kappa r) r d r=\frac{1}{\kappa^{2}} \int_{0}^{a / \kappa} J_{0}(r) r d r=\frac{a}{\kappa^{3}} J_{1}(a / \kappa) .
$$

For $a=1$ :

$$
\int_{0}^{1} J_{0}(\kappa r) r d r=\frac{1}{\kappa^{3}} J_{1}(1 / \kappa)=\frac{J_{1}\left(\frac{1}{\epsilon_{n}(0)}\right)}{\epsilon_{n}^{3}(0)},
$$

plugging it in (36) gives the expression for $a_{n}$

Generally, orthonormal basis consists of the functions:

$$
\Sigma_{\nu}(\rho)=\frac{1}{\sqrt{N_{01}}} J_{\nu}\left(\sqrt{\lambda_{\nu, m}} \rho\right)=\frac{1}{\sqrt{N_{01}}} J_{\nu}\left(\frac{\epsilon_{m}(\nu)}{\rho_{0}} \rho\right) .
$$

For the equation (26), the relation (29) account yields:

$$
Z_{t}+\alpha W_{t}+J\left(-\lambda W+W_{z z}\right)=0 .
$$

Next, similarly, (23) reads:

$$
\alpha Z_{t}+K Z+J\left(-\lambda Z+Z_{z z}\right)=W_{t} .
$$

Multiplying (41) by $\alpha$ and subtracting, we have:

$$
\alpha^{2} W_{t}+J \alpha\left(-\lambda W+W_{z z}\right)-K Z-J\left(-\lambda Z+Z_{z z}\right)=-W_{t} .
$$

Next, multiplying (42) by $\alpha$ and adding, gives:

$$
\left(1+\alpha^{2}\right) Z_{t}+J\left(-\lambda W+W_{z z}\right)+\alpha K Z+\alpha J\left(-\lambda Z+Z_{z z}\right)=0 .
$$

Finally, we arrive at the system:

$$
Z_{t}=\frac{J \lambda W-K Z \alpha-J W_{z z}-J \alpha Z_{z z}+J Z \alpha \lambda}{1+\alpha^{2}}, \quad W_{t}=\frac{J Z_{z z}+K Z-J Z \lambda-J \alpha W_{z z}+J \alpha \lambda W}{1+\alpha^{2}},
$$

which constitutes the mode evolution system. In matrix form it reads:

$$
\begin{aligned}
& \left(\begin{array}{c}
Z \\
W
\end{array}\right)_{t}= \\
& \frac{J}{1+\alpha^{2}}\left(\begin{array}{cc}
-\frac{K}{J} \alpha-\alpha \partial_{z z}+\alpha \lambda & \lambda-\partial_{z z} \\
\partial_{z z}+\frac{K}{J}-\lambda & -\alpha \partial_{z z}+\alpha \lambda
\end{array}\right)\left(\begin{array}{c}
Z \\
W
\end{array}\right) \text {. }
\end{aligned}
$$


After Fourier transformation by z, in k-representation $Z \rightarrow \tilde{Z}, \ldots$, the evolution in $k$-domain is described by:

$$
\left(\begin{array}{c}
\tilde{Z} \\
\tilde{W}
\end{array}\right)_{t}=\frac{J}{1+\alpha^{2}}\left(\begin{array}{cc}
-w \alpha+\alpha k^{2}+\alpha \lambda & \lambda+k^{2} \\
-k^{2}+w-\lambda & \alpha k^{2}+\alpha \lambda
\end{array}\right)\left(\begin{array}{c}
\tilde{Z} \\
\tilde{W}
\end{array}\right)
$$

where it is denoted: $w=\frac{K}{J}$.

\subsection{On dispersion/attenuation}

4.4.1. Dispersion equation. The dispersion equation reads:

$$
\operatorname{det}\left(\begin{array}{cc}
-w \alpha+\alpha k^{2}+\alpha \lambda-i \omega & \lambda+k^{2} \\
-k^{2}+w-\lambda & \alpha k^{2}+\alpha \lambda-i \omega
\end{array}\right)=0 .
$$

Let us denote $\lambda+k^{2}=\mu, \alpha \mu-i \omega=\varkappa$, having:

$$
\operatorname{det}\left(\begin{array}{cc}
-w \alpha+\varkappa & \mu \\
-\mu+w & \varkappa
\end{array}\right)=0
$$

Solving the equation (47) with respect to $\omega$, gives dispersion branches $\omega_{ \pm}$.

4.4.2. Dispersion branches. The roots of the quadratic Eq. (47) are expressed as:

$$
\omega_{ \pm}= \pm \frac{i}{2} \sqrt{4 w\left(k^{2}+\lambda\right)+w^{2} \alpha^{2}-4\left(k^{2}+\lambda\right)^{2}}-\frac{1}{2} i \alpha\left(k^{2}-w+\lambda\right) .
$$

If $4 w\left(k^{2}+\lambda\right)+w^{2} \alpha^{2}-4\left(k^{2}+\lambda\right)^{2}>0$, the solution oscillates. The $\omega_{ \pm}$are complex if:

$$
4 w\left(k^{2}+\lambda\right)+w^{2} \alpha^{2}-4\left(k^{2}+\lambda\right)^{2}<0,
$$

which gives damping contributions, eventual cut of guide propagation. Otherwise, the borders of the range are defined by:

the condition (49) holds if either

$$
4 w\left(k^{2}+\lambda\right)+w^{2} \alpha^{2}-4\left(k^{2}+\lambda\right)^{2}=0
$$

$$
k^{2}<\frac{w}{2}\left(1-\sqrt{\alpha^{2}+1}\right)-\lambda<0
$$

which is impossible for real $\mathrm{k}$, or:

$$
k^{2}>\frac{w}{2}\left(1+\sqrt{\alpha^{2}+1}\right)-\lambda
$$

\subsection{Dynamical projectors}

4.5.1. Evolution operator in k-domain. Returning to original form of the evolution operator:

$$
L_{k}=\frac{J}{1+\alpha^{2}}\left(\begin{array}{cc}
-\frac{K}{J} \alpha+\alpha k^{2}+\alpha \lambda & \lambda+k^{2} \\
-k^{2}+\frac{K}{J}-\lambda & \alpha k^{2}+\alpha \lambda
\end{array}\right),
$$

denoting, as before, $w=\frac{K}{J}, \lambda+k^{2}=\mu$, we arrive to more compact form, omitting the constant factor, that do not contribute in the projectors ( to eigen subspaces of $L_{k}$ ) expressions. We write:

$$
L_{k}^{\prime}=\left(\begin{array}{cc}
-w \alpha+\alpha \mu & \mu \\
w-\lambda & \alpha \mu
\end{array}\right)
$$

that enters the algorithm below, see the key formula (57). 
4.5.2. Eigenvector matrix. The eigenvectors of (51) with unit lower component are evaluated as:

$$
\begin{aligned}
& \left\{\begin{array}{c}
\frac{1}{2 \mu-2 w}\left(w \alpha+\sqrt{w^{2} \alpha^{2}+4 w \mu-4 \mu^{2}}\right) \\
1
\end{array}\right\} \leftrightarrow \alpha \mu-\frac{1}{2} w \alpha-\frac{1}{2} \sqrt{w^{2} \alpha^{2}+4 w \mu-4 \mu^{2}} \\
& \left\{\begin{array}{c}
\frac{1}{2 \mu-2 w}\left(w \alpha-\sqrt{w^{2} \alpha^{2}+4 w \mu-4 \mu^{2}}\right) \\
1
\end{array}\right\} \leftrightarrow \alpha \mu-\frac{1}{2} w \alpha+\frac{1}{2} \sqrt{w^{2} \alpha^{2}+4 w \mu-4 \mu^{2}} .
\end{aligned}
$$

Denoting:

$$
q^{2}=4 w\left(k^{2}+\lambda\right)+w^{2} \alpha^{2}-4\left(k^{2}+\lambda\right)^{2}, \quad p=2 \mu-2 w
$$

we write the eigenmatrix as:

$$
\Psi=\left(\begin{array}{cc}
\frac{1}{p}(w \alpha+q) & \frac{1}{p}(w \alpha-q) \\
1 & 1
\end{array}\right)
$$

4.5.3. Algorithm of projectors construction. Denoting $\tilde{\Lambda}=\operatorname{diag}\left\{\lambda_{i}\right\}$ the diagonal eigenvalue matrix, $\Psi$ - eigenmatrix, we transform the eigenproblem in $k$-domain:

$$
\tilde{L} \Psi=\Psi \tilde{\Lambda}
$$

that is, by the way, the definition of $\Psi$, too:

$$
\tilde{L}=\Psi \tilde{\Lambda} \Psi^{-1}
$$

or, in components, it gives the spectral decomposition of the matrix $\tilde{L}$ :

$$
\tilde{L}_{i j}=\Psi_{i k} \tilde{\Lambda}_{k l} \Psi_{l j}^{-1}=\Psi_{i k} \lambda_{k} \Psi_{k j}^{-1}=\sum_{k} \lambda_{k} \Psi_{i k} \Psi_{k j}^{-1}=\sum_{s} \lambda_{s}\left(\tilde{P}^{s}\right)_{i j}
$$

Where $\tilde{P}^{s}$ are the $k$-transforms of the dynamic projecting operators [27]:

$$
\left(\tilde{P}^{s}\right)_{i j}=\Psi_{i s} \Psi_{s j}^{-1}
$$

with the inverse to (53):

$$
\Psi^{-1}=\left(\begin{array}{cc}
\frac{1}{2} \frac{p}{q} & \frac{1}{2 q}(q-w \alpha) \\
-\frac{1}{2} \frac{p}{q} & \frac{1}{2 q}(q+w \alpha)
\end{array}\right)
$$

Reading the relation (57), let us do the first step of the first projector construction in the form:

$$
P^{1}=\left(\begin{array}{c}
\frac{w \alpha+q}{p} \\
1
\end{array}\right)\left(\begin{array}{cc}
\frac{1}{2} \frac{p}{q} & \frac{1}{2 q}(q-w \alpha)
\end{array}\right)=\left(\begin{array}{cc}
\frac{1}{2 q}(q+w \alpha) & \frac{1}{2 p q}(q+w \alpha)(q-w \alpha) \\
\frac{1}{2} \frac{p}{q} & \frac{1}{2 q}(q-w \alpha)
\end{array}\right) .
$$

Acting by the projector $P^{1}$ to the basic vector gives:

$$
\begin{gathered}
\left(\begin{array}{cc}
\frac{1}{2 q}(q+w \alpha) & \frac{1}{2 p q}(q+w \alpha)(q-w \alpha) \\
\frac{1}{2} \frac{p}{q} & \frac{1}{2 q}(q-w \alpha)
\end{array}\right)\left(\begin{array}{c}
Z \\
W
\end{array}\right)= \\
\left(\begin{array}{c}
\frac{1}{2} \frac{Z}{q}(q+w \alpha)+\frac{1}{2 p q} W(q+w \alpha)(q-w \alpha) \\
\frac{1}{2} Z \frac{p}{q}+\frac{1}{2 q} W(q-w \alpha)
\end{array}\right)=\left(\begin{array}{c}
\frac{(q+w \alpha)}{p} \Pi \\
\Pi
\end{array}\right),
\end{gathered}
$$

where the notation for the new field $\Pi$ is introduced:

$$
\Pi=\frac{p}{2 q} Z+\frac{q-w \alpha}{2 q} W .
$$


Going to the second step, in the direct analogy, we have:

$$
P^{2}=\left(\begin{array}{c}
\frac{w \alpha-q}{p} \\
1
\end{array}\right)\left(\begin{array}{cc}
-\frac{1}{2} \frac{p}{q} & \frac{1}{2 q}(q+w \alpha)
\end{array}\right)=\left(\begin{array}{cc}
\frac{1}{2 q}(q-w \alpha) & -\frac{1}{2 p q}(q+w \alpha)(q-w \alpha) \\
-\frac{1}{2} \frac{p}{q} & \frac{1}{2 q}(q+w \alpha)
\end{array}\right)
$$

acting by the projector $P^{2}$ to the basic vector gives:

$$
\begin{gathered}
\left(\begin{array}{cc}
\frac{1}{2 q}(q-w \alpha) & -\frac{1}{2 p q}(q+w \alpha)(q-w \alpha) \\
-\frac{1}{2} \frac{p}{q} & \frac{1}{2 q}(q+w \alpha)
\end{array}\right)\left(\begin{array}{c}
Z \\
W
\end{array}\right)= \\
\left(\begin{array}{c}
\frac{1}{2} \frac{Z}{q}(q-w \alpha)-\frac{1}{2 p q} W(q+w \alpha)(q-w \alpha) \\
\frac{q+w \alpha}{2 q} W-\frac{1}{2} Z \frac{p}{q}
\end{array}\right)=\left(\begin{array}{c}
\frac{(q-w \alpha)}{p} \Lambda \\
\Lambda
\end{array}\right),
\end{gathered}
$$

where the second field:

$$
\Lambda=\frac{q+w \alpha}{2 q} W-\frac{p}{2 q} Z
$$

is introduced. The inverse to $(58,59)$ reads as:

$$
\begin{gathered}
W=\Pi+\Lambda, \\
Z=\frac{1}{p}(-q \Lambda+q \Pi+w \alpha \Lambda+w \alpha \Pi) .
\end{gathered}
$$

4.5.4. Evolution dynamics splitting. Returning to the complete form of the evolution operator:

$$
L_{k}=\frac{J}{1+\alpha^{2}}\left(\begin{array}{cc}
-w \alpha+\alpha \mu & \mu \\
-\mu+w & \alpha \mu
\end{array}\right)
$$

we define the evolution vector as:

$$
\phi=\left(\begin{array}{c}
Z \\
W
\end{array}\right)
$$

having:

$$
\phi_{t}=L_{k} \phi
$$

To split the problem, let us act by operators $P^{1,2}$ to Eq. (62), that commute with $L_{k}$. Hence. on a way to the variable $\Pi$ we obtain:

while the right side for $s=1$ gives:

$$
P^{s} \phi_{t}=L_{k} P^{s} \phi
$$

$$
\begin{aligned}
L_{k} P^{1} \phi=\frac{J}{1+\alpha^{2}}\left(\begin{array}{cc}
-w \alpha+\alpha \mu & \mu \\
-\mu+w & \alpha \mu
\end{array}\right)\left(\begin{array}{c}
\frac{(q+w \alpha)}{p} \Pi \\
\Pi
\end{array}\right)= \\
\frac{J}{1+\alpha^{2}}\left(\begin{array}{c}
\mu \Pi-\frac{1}{p} \Pi(w \alpha-\alpha \mu)(q+w \alpha) \\
\alpha \mu \Pi+\frac{1}{p} \Pi(w-\mu)(q+w \alpha)
\end{array}\right)
\end{aligned}
$$

Reading the second line, we see, that:

$$
\Pi_{t}=\frac{J}{1+\alpha^{2}} \frac{-q \mu+w^{2} \alpha+q w+p \alpha \mu-w \alpha \mu}{p} \Pi=\beta \Pi,
$$

where:

$$
p=2 k^{2}-2 w+2 \lambda, \quad \mu=\lambda+k^{2}, \quad \beta=J \frac{-q \mu+w^{2} \alpha+q w+p \alpha \mu-w \alpha \mu}{p\left(1+\alpha^{2}\right)} .
$$

Finally, the function:

$$
\Pi=C \exp (\beta t)
$$


solves the equation (63). Similar, for $\Lambda$-state, we evaluate:

$$
L_{k} P^{2} \phi=\frac{J}{1+\alpha^{2}}\left(\begin{array}{cc}
-w \alpha+\alpha \mu & \mu \\
-\mu+w & \alpha \mu
\end{array}\right)\left(\begin{array}{c}
-\frac{(q-w \alpha)}{p} \Lambda \\
\Lambda
\end{array}\right)=
$$

$$
\left(\begin{array}{c}
J \Lambda \frac{\mu}{\alpha^{2}+1}+J \Lambda(w \alpha-\alpha \mu) \frac{q-w \alpha}{\alpha^{2}+1} \\
J \alpha \Lambda \frac{\mu}{\alpha^{2}+1}-\frac{J}{p} \Lambda(w-\mu) \frac{q-w \alpha}{\alpha^{2}+1}
\end{array}\right)
$$

which gives:

$$
J \alpha \Lambda \frac{\mu}{\alpha^{2}+1}-\frac{J}{p} \Lambda(w-\mu) \frac{q-w \alpha}{\alpha^{2}+1}=\frac{J}{p} \frac{(q+p \alpha) \mu+w(\alpha w-q-\alpha \mu)}{\alpha^{2}+1} \Lambda,
$$

arriving at the equation:

$$
\Lambda_{t}=\frac{J}{p} \frac{(q+p \alpha) \mu+w(\alpha w-q-\alpha \mu)}{\alpha^{2}+1} \Lambda=\eta \Lambda,
$$

and its solution:

$$
\Lambda=D \exp (\eta t)
$$

where:

$$
\eta=J \frac{(q+p \alpha) \mu+w(\alpha w-q-\alpha \mu)}{p\left(\alpha^{2}+1\right)} .
$$

\subsection{The solution of (45) pick up}

4.6.1. One-term $\Omega^{\prime}$. For the reader's convenience, we reproduce the result of Fourier one-term contribution via: (25): $\Sigma(\rho)=N_{\nu, m}^{-1 / 2} J_{\nu}\left(\frac{\epsilon_{m}(\nu)}{\rho_{0}} \rho\right), \Omega^{\prime}=R+i S$. We further skip the normalizing factor and c.c. term, having

$$
R(\varphi, \rho, z, t)=\exp [i \nu \varphi] J_{\nu}\left(\frac{\epsilon_{m}(\nu)}{\rho_{0}} \rho\right) Z(z, t), S=\exp [i \nu \varphi] J_{\nu}\left(\frac{\epsilon_{m}(\nu)}{\rho_{0}} \rho\right) W(z, t),
$$

adding results yields:

$$
\Omega_{\nu m}^{\prime}(k)=R+i S=\exp [i \nu \varphi] J_{\nu}\left(\epsilon_{m}(\nu) \frac{\rho}{\rho_{0}}\right)(Z+i W) .
$$

Going to the $\Pi, \Lambda$ variables, we transform:

$$
Z+i W=\left(\frac{q+w \alpha}{p}+i\right) C \exp (\beta t)+\left(\frac{-q+w \alpha}{p}+i\right) D \exp (\eta t) .
$$

4.6.2. Final expression. Taking the expressions for parameters (52), we rewrite:

$$
\begin{gathered}
q=\sqrt{w^{2} \alpha^{2}+4 k^{2} w-4 k^{4}-4 \frac{\epsilon_{m}^{4}}{\rho_{0}^{4}}+4 w \frac{\epsilon_{m}^{2}}{\rho_{0}^{2}}-8 k^{2} \frac{\epsilon_{m}^{2}}{\rho_{0}^{2}}}, \\
p=2 k^{2}-2 w+2 \lambda=2 k^{2}-2 w+2 \frac{\epsilon_{m}^{2}(\nu)}{\rho_{0}^{2}}
\end{gathered}
$$

which should be plugged in:

$$
\begin{aligned}
& \beta=J \frac{\mu(p \alpha-q-w \alpha)+w^{2} \alpha+q v}{p\left(1+\alpha^{2}\right)}, \\
& \eta=J \frac{\mu(p \alpha+q-w \alpha)+w^{2} \alpha-q w}{p\left(1+\alpha^{2}\right)} .
\end{aligned}
$$

For the one-(transversal)-mode solution at $z$-domain we obtain:

$$
\begin{aligned}
& \Omega_{\nu m}^{\prime}(z, t)= \\
& \quad N_{\nu, m}^{-1 / 2} J_{\nu}\left(\epsilon_{m}(\nu) \frac{\rho}{\rho_{0}}\right) e^{i \nu \phi} \int d k e^{-i k z}\left(\left(\frac{w \alpha+q}{p}+i\right) C e^{\beta t}+\left(\frac{w \alpha-q}{p}+i\right) D e^{\eta t}\right)+\text { c.c. }
\end{aligned}
$$




\section{Two problems}

\subsection{Initiation of $D W$ in experiment}

A comprehensive review on nanowires and nanotubes physics was published recently [28, 29]. It contains a description of the synthesis, magnetic properties and applications of magnetic cylindrical nanowires and nanotubes, including single domain wall statics and dynamics, with very rich set of references. By "nano" the authors conventionally consider diameters reasonably smaller than a micrometer. One possibility to govern magnetoresistence by light is studied theoretically in [30]. In a real experiments a DW creation is realized either in the vicinity of a distant point from the ends [5] or at the end point vicinity [31]. In the first case, the DW is excited by a few-turns coin, the measurement of its velocity is performed by similar coins shifted left and right, that pick up Faraday pulses. A similar approach is used to measure the velocity in the second case as well, as it allows one to estimate the DW length via the coin voltage form [31], The measurements of DW velocity exhibits conventionally a linear dependence on magnetic field [32], however the presence of magnetic inhomogeneities forces a DW acceleration [5, 25].

A statement of problem at a vicinity of a wire end implies account of so-called "stray field" [23].

\subsection{Cauchy problem for a DW excitation far from ends}

Let us consider the simplest case of complete cylindrical symmetry of solution, i.e. $\phi$-independent solution, that fixes the case $\nu=0$, taking the first Bessel mode, which determines the radial dependence.

As an initial condition, let us choose the delta-impulse posed at the $z=0$ point, chosen far from a wire ends. It mimics the excitation of the DW pair by a current pulse at a few-turn coin. Omitting the Bessel function factor $J_{0}\left(\epsilon_{1}(0) \frac{\rho}{\rho_{0}}\right)$ at $(70)$, we impose the following condition:

$$
\Omega_{01}^{\prime \prime}(z, 0)=\int d k \exp [-i k z]\left(\left(\frac{q+w \alpha}{p}+i\right) C+\left(\frac{-q+w \alpha}{p}+i\right) D\right)=\delta(z) .
$$

It yields:

$$
\begin{gathered}
\int d z \exp \left[i k^{\prime} z\right] \int d k \exp [-i k z]\left(\left(\frac{q+w \alpha}{p}+i\right) C+\left(\frac{-q+w \alpha}{p}+i\right) D\right)=\int d z \exp \left[i k^{\prime} z\right] \delta(z)=1 \\
\left(\left(\frac{q(k)+w \alpha}{p(k)}+i\right) C+\left(\frac{-q(k)+w \alpha}{p(k)}+i\right) D\right)=1
\end{gathered}
$$

It gives the following relations:

1. Zero imaginary part, that gives $D=-C$.

2. The real one reads: $C \frac{2 q(k)}{p(k)}=1$, or $C(k)=\frac{p(k)}{2 q(k)}$.

Then, plugging the results into the relation (70), we arrive at:

$$
\Omega_{01}^{\prime \prime}(z, t)=\frac{1}{2} \int d k \exp [-i k z]\left[\left(\frac{q+w \alpha}{q}+i \frac{p}{q}\right) \exp (\beta t)-\left(\frac{-q+w \alpha}{q}+i \frac{p}{q}\right) \exp (\eta t)\right] .
$$

Let us introduce new parameters: $A=\frac{J \alpha}{1+\alpha^{2}} \frac{\mu(p-w)+w^{2}}{p}, B=\frac{J q}{1+\alpha^{2}} \frac{-\mu \alpha+w}{p}$, having:

$$
\beta=A+B, \quad \eta=A-B \text {. }
$$

Finally, in new notations,

$$
\Omega_{01}^{\prime \prime}(z, t)=\frac{1}{2} \int d k \exp [-i k z+A t]\left[\left(\frac{w \alpha}{q}+1+i \frac{p}{q}\right) \exp (B t)-\left(\frac{w \alpha}{q}-1+i \frac{p}{q}\right) \exp (-B t)\right] .
$$

The parameters in the approximation $\alpha \ll 1$, simplify as:

$$
\begin{gathered}
q=w \sqrt{\alpha^{2}+4 \frac{k^{2}}{w}-4\left(\frac{k^{2}}{w}\right)^{2}}, \\
\mu=k^{2}+\lambda_{0,1}, \quad \frac{p}{2 w}=\frac{k^{2}}{w}+\frac{\lambda_{0.1}}{w}-1, \\
A \approx J \alpha \frac{\left(k^{2}+\lambda_{0,1}\right)\left(2 k^{2}-3 w+2 \lambda_{0.1}\right)+w^{2}}{2 k^{2}-2 w+2 \lambda_{0.1}}, \\
B \approx J q \frac{-\mu \alpha+w}{p} .
\end{gathered}
$$

The linearity of the equations allows one to build more general solution as the mode superposition. 


\subsection{DW dynamics, forced by magnetic field}

5.3.1. Inhomogeneous system. Basic linear complex in-homogeneous equation is written as:

$$
\mathcal{L} \Omega^{l}=(\alpha+i) \Omega_{t}^{l}+J \nabla^{2} \Omega^{l}+K \Re \Omega^{l}=\gamma H,
$$

where: $\Omega^{l}=R^{l}+i S^{l}$, that, adding and subtracting results for real and imaginary parts, yields:

$$
\begin{gathered}
\left(\alpha+\frac{1}{\alpha}\right) S_{t}^{l}+J \nabla^{2} S^{l}-\frac{1}{\alpha} K R^{l}+\frac{J}{\alpha} \nabla^{2} R^{l}=-\frac{\gamma}{\alpha} H, \\
\left(\alpha+\frac{1}{\alpha}\right) R_{t}^{l}+K R^{l}+J \nabla^{2} R^{l}+\frac{1}{\alpha} J \nabla^{2} S^{l}=\gamma H .
\end{gathered}
$$

Simplifying for the case of small relaxation term, $\alpha \ll 1$, we arrive at the system:

$$
\begin{gathered}
R_{t}^{l}+\alpha K R^{l}+\alpha J \nabla^{2} R^{l}+J \nabla^{2} S^{l}=\alpha \gamma H, \\
S_{t}^{l}+\alpha J \nabla^{2} S^{l}-K R^{l}-J \nabla^{2} R^{l}=-\gamma H .
\end{gathered}
$$

Next, let us conventionally transform it to the matrix notation:

$$
\left(\begin{array}{c}
R^{l} \\
S^{l}
\end{array}\right)_{t}+\left(\begin{array}{cc}
\alpha K+\alpha J \nabla^{2} & J \nabla^{2} \\
-K-J \nabla^{2} & \alpha J \nabla^{2}
\end{array}\right)\left(\begin{array}{c}
R^{l} \\
S^{l}
\end{array}\right)=\left(\begin{array}{c}
\alpha \gamma H \\
-\gamma H
\end{array}\right) .
$$

The general solution of the inhomogeneous system (c.c. and normalizing factor omitted) is given by two equalities:

$$
\begin{gathered}
R^{l}(\varphi, \rho, z, t)=\sum_{\nu, m} \exp [i \nu \varphi] J_{\nu}\left(\epsilon_{m}(\nu) r\right) Z_{m \nu}^{l}(z, t), \\
S^{l}=\sum_{\nu, m} \exp [i \nu \varphi] J_{\nu}\left(\epsilon_{m}(\nu) r\right) W_{m \nu}^{l}(z, t) .
\end{gathered}
$$

Putting unit expansion at r.h.s., for example, for a given $\nu$ :

$$
H(z, t)=\sum_{m=1} a_{m \nu}(z, t) J_{\nu}\left(\epsilon_{m}(\nu) r\right) \exp [i \nu \varphi],
$$

we arrive at the two-component inhomogeneous system, transformed to matrix form. For such a transverse mode, the system reads:

$$
\begin{aligned}
\left(\begin{array}{c}
Z_{m \nu}^{l}(z, t) \\
W_{m \nu}^{l}(z, t)
\end{array}\right)_{t}+\alpha J\left(\begin{array}{cc}
w-\lambda_{m \nu}+\partial_{z z} & \frac{1}{\alpha}\left(-\lambda_{m \nu}+\partial_{z z}\right) \\
\lambda_{m \nu}-\partial_{z z}-\frac{w}{\alpha} & -\lambda_{m \nu}+\partial_{z z}
\end{array}\right)\left(\begin{array}{c}
Z_{m \nu}^{l}(z, t) \\
W_{m \nu}^{l}(z, t)
\end{array}\right)= & \\
& \left(\begin{array}{c}
\alpha \gamma a_{m \nu}(z, t) \\
-\gamma a_{m \nu}(z, t)
\end{array}\right) .
\end{aligned}
$$

5.3.2. Inhomogeneous system projecting. Now, it is convenient to apply projecting operators, in $k$-domain, putting:

$$
a_{m \nu}(z, t)=\int d k \exp [-i k z] \widetilde{a}_{m \nu}(k, t),
$$

and, similar to homogeneous case, it is written:

$$
\left(\begin{array}{c}
\widetilde{Z}_{m \nu}^{l}(k, t) \\
\widetilde{W}_{m \nu}^{l}(k, t)
\end{array}\right)_{t}+\alpha J\left(\begin{array}{cc}
w-\lambda_{m \nu}-k^{2} & \frac{1}{\alpha}\left(-\lambda_{m \nu}-k^{2}\right) \\
\lambda_{m \nu}+k^{2}-\frac{w}{\alpha} & -\lambda_{m \nu}-k^{2}
\end{array}\right)\left(\begin{array}{c}
\widetilde{Z}_{m \nu}^{l}(k, t) \\
\widetilde{W}_{m \nu}^{l}(k, t)
\end{array}\right)=\left(\begin{array}{c}
\alpha \gamma \widetilde{a}_{m \nu}(k, t) \\
-\gamma \widetilde{a}_{m \nu}(k, t)
\end{array}\right) .
$$

Denoting again, $\lambda_{m \nu}+k^{2}=\mu$, we simplify the evolution operator for the approximate system as:

$$
L=\alpha J\left(\begin{array}{cc}
w-\mu & -\frac{\mu}{\alpha} \\
\mu-\frac{w}{\alpha} & -\mu
\end{array}\right) .
$$

Let us show Maple-made eigenvectors and eigenvalues: 


$$
\begin{aligned}
& \left\{\begin{array}{c}
-\frac{w \alpha-\sqrt{w^{2} \alpha^{2}+4 w \mu-4 \alpha \mu^{2}}}{2 w-2 \alpha \mu} \\
1
\end{array}\right\} \leftrightarrow \frac{1}{2 \alpha^{2}}\left(w \alpha^{2}-2 \alpha^{2} \mu\right)-\frac{1}{2 \alpha} \sqrt{w^{2} \alpha^{2}+4 w \mu-4 \alpha \mu^{2}}, \\
& \left\{\begin{array}{c}
-\frac{w \alpha+\sqrt{w^{2} \alpha^{2}+4 w \mu-4 \alpha \mu^{2}}}{2 w-2 \alpha \mu} \\
1
\end{array}\right\} \leftrightarrow \frac{1}{2 \alpha} \sqrt{w^{2} \alpha^{2}+4 w \mu-4 \alpha \mu^{2}}+\frac{1}{2 \alpha^{2}}\left(w \alpha^{2}-2 \alpha^{2} \mu\right) .
\end{aligned}
$$

Going to the more compact form, we denote:

$$
-\frac{w \alpha}{2 w-2 \alpha \mu}\left(1-\sqrt{1+\frac{4 \mu}{w \alpha^{2}}-\frac{4 \mu^{2}}{w^{2} \alpha}}\right)=r(1-s),
$$

where:

$$
r=-\frac{w \alpha}{2 w-2 \alpha \mu}, \quad s=\sqrt{1+\frac{4 \mu}{w \alpha^{2}}-\frac{4 \mu^{2}}{w^{2} \alpha}} .
$$

5.3.3. The projectors and its application. The projector matrices are built by formula: (57) on base of the eigenmatrix of the evolution operator $L$, see (77)

$$
\Psi=\left(\begin{array}{cc}
r(1-s) & r(1+s) \\
1 & 1
\end{array}\right)
$$

and its inverse:

We write both projecting matrices as:

$$
\left(\begin{array}{cc}
-\frac{1}{2 r s} & \frac{1}{2 s}(s+1) \\
\frac{1}{2 r s} & \frac{1}{2 s}(s-1)
\end{array}\right)
$$

$$
\begin{aligned}
& \pi_{1}=\left(\begin{array}{c}
r(1-s) \\
1
\end{array}\right)\left(\begin{array}{cc}
-\frac{1}{2 r s} & \frac{1}{2 s}(s+1)
\end{array}\right)=\left(\begin{array}{cc}
\frac{1}{2 s}(s-1) & -\frac{1}{2} \frac{r}{s}\left(s^{2}-1\right) \\
-\frac{1}{2 r s} & \frac{1}{2 s}(s+1)
\end{array}\right), \\
& \pi_{2}=\left(\begin{array}{c}
r(1-s) \\
1
\end{array}\right)\left(\begin{array}{cc}
\frac{1}{2 r s} & \frac{1}{2 s}(s-1)
\end{array}\right)=\left(\begin{array}{cc}
-\frac{1}{2 s}(s-1) & -\frac{1}{2} \frac{r}{s}(s-1)^{2} \\
\frac{1}{2 r s} & \frac{1}{2 s}(s-1)
\end{array}\right) .
\end{aligned}
$$

The action of $\pi_{1,2}$ to the matrix equation (76), using the shorthand for the evolution vector:

$$
\left(\begin{array}{c}
\widetilde{Z} \\
\widetilde{W}
\end{array}\right)
$$

gives:

$$
\alpha J\left(\begin{array}{cc}
w-\mu & -\frac{\mu}{\alpha} \\
\mu-\frac{w}{\alpha} & -\mu
\end{array}\right)\left(\begin{array}{cc}
\frac{1}{2 s}(s-1) & -\frac{1}{2} \frac{r}{s}\left(s^{2}-1\right) \\
-\frac{1}{2 r s} & \frac{1}{2 s}(s+1)
\end{array}\right)\left(\begin{array}{c}
\widetilde{Z} \\
\widetilde{W}
\end{array}\right)=
$$

The conventional notation:

$$
\left(\begin{array}{c}
\frac{1}{2 s} \widetilde{Z}(s-1)-\frac{1}{2} \frac{r}{s} \widetilde{W}\left(s^{2}-1\right) \\
\frac{1}{2 s} \widetilde{W}(s+1)-\frac{1}{2 r s} \widetilde{Z}
\end{array}\right)
$$

$$
\frac{(s+1)}{2 s} \widetilde{W}-\frac{1}{2 r s} \widetilde{Z}=\pi,
$$

gives for the upper component, proportional to $\pi$ : $-(s-1) r \pi$. The projecting operator at $z$-domain is the integral operator with the kernel, that is expressed via $r, s$, as well as the formula for $\pi$ is built as integral transform [27]. 
Approximate equations for long wave range (small $k$ ) is obtained by expansion the matrix elements of $\pi$ in $k$-power series.

\subsection{Relaxation equation in $k$-domain}

The result in terms of the new variable $\pi$, see (79), we obtain, introducing the shorthand $\widetilde{a}_{m \nu}(k, t)=a$, whence the equation (76) projection reads as:

$$
\begin{aligned}
& \alpha J\left(\begin{array}{cc}
w-\mu & -\frac{\mu}{\alpha} \\
\mu-\frac{w}{\alpha} & -\mu
\end{array}\right)\left(\begin{array}{c}
-(s-1) r \pi \\
\pi
\end{array}\right)=\left(\begin{array}{c}
-\pi J \mu-\pi J r \alpha(w-\mu)(s-1) \\
-\pi J \alpha \mu-\pi J r \alpha\left(\mu-\frac{w}{\alpha}\right)(s-1)
\end{array}\right)= \\
&\left(\begin{array}{cc}
\frac{1}{2 s}(s-1) & -\frac{1}{2} \frac{r}{s}(s-1)(s+1) \\
-\frac{1}{2 r s} & \frac{1}{2 s}(s+1)
\end{array}\right)\left(\begin{array}{c}
\alpha \gamma a \\
-\gamma a
\end{array}\right)= \\
&\left(\begin{array}{c}
\frac{1}{2} \frac{a}{q} \alpha \gamma(s-1)+\frac{1}{2} a \frac{r}{s} \gamma(s-1)(s+1) \\
-\frac{1}{2} \frac{a}{s} \gamma(s+1)-\frac{1}{2} \frac{a}{r s} \alpha \gamma
\end{array}\right) .
\end{aligned}
$$

Plugging the expression for $\pi$ (79) one arrives at the relaxation equation:

$$
\pi_{t}+J(-\alpha \mu-s w+s \alpha \mu+r s w-r s \alpha \mu) \pi=-\frac{1}{2} \gamma \frac{r+\alpha+r s}{s r} a .
$$

Denoting:

$$
o=J(-\alpha \mu-s w+s \alpha \mu+r s w-r s \alpha \mu), \quad h=-\frac{1}{2} \gamma \frac{r+\alpha+r s}{r s} a,
$$

where $h$ is proportional to magnetic field, see (74). We transform:

$$
\pi_{t}+o \pi=h, \quad e^{-o t}\left(\pi e^{o t}\right)_{t}=h,
$$

having the solution for time-dependent homogeneous magnetic field switching at $k$-domain:

$$
\pi e^{o t}=\int_{0}^{t} h(\tau) e^{o \tau} d \tau, \quad \pi=e^{-o t} \int_{0}^{t} h(\tau) e^{o \tau} d \tau .
$$

For the constant magnetic field $h(\tau)=h \theta(\tau)$ and the zero initial condition gives:

$$
\pi=e^{-o t} \int_{0}^{t} h e^{o \tau} d \tau=\frac{h}{o}\left(1-e^{-o t}\right),
$$

then, for a real $o, o^{-1}$ is relaxation time of $k$-mode.

5.4.1. Back to the $z$-domain. The inverse Fourier transform gives:

$$
\Pi_{m \nu}(z, t)=\frac{h}{2 \pi} \int d k \exp [-i k z] \frac{\left(1-e^{-o(k) t}\right)}{o(k)} .
$$

Plugging $r=-\frac{w \alpha}{2 w-2 \alpha \mu}, \mu=\lambda_{m \nu}+k^{2}$ into $o$, we obtain the frequency:

$$
o=-\frac{1}{2} J \alpha\left(-w+2 \lambda_{m \nu}+s w+2 k^{2}\right) .
$$

The dependence of $o$ on $k^{2}$ for real $s$ is presented at Fig. 1, and, at the range of imaginary $s$ at Fig. 2. The "left" variable is introduced in similar way via the projecting by $\pi_{2}$. 


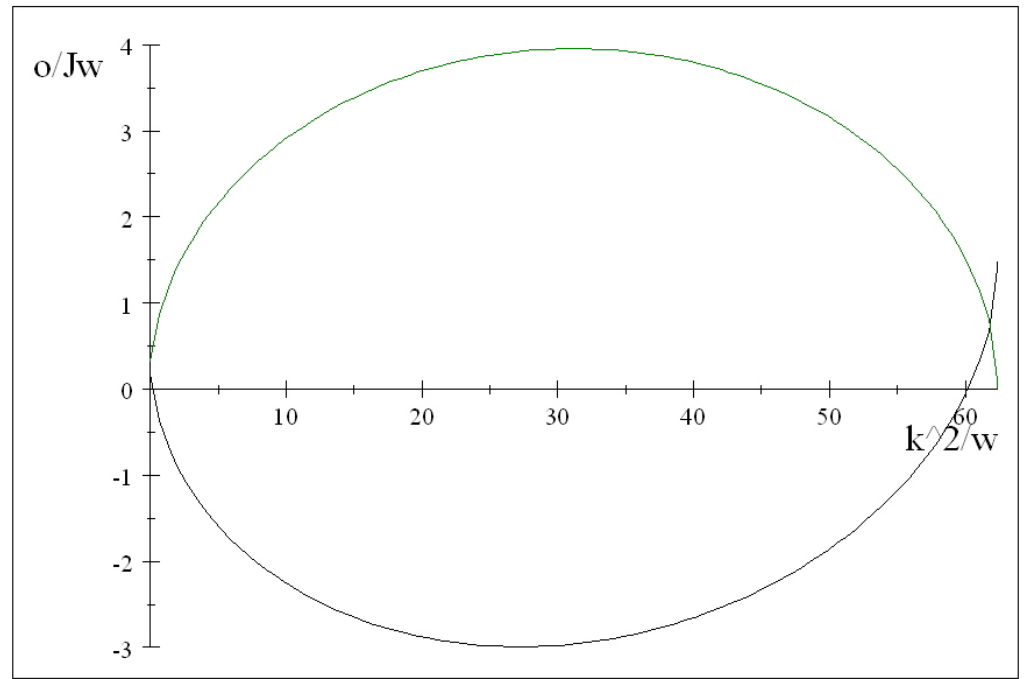

FIG. 1. The dependence of $o / K$ on $k^{2} / w$ (black) is presented at range of real root expression (green) for the values of parameters $\nu=0, m=1$.

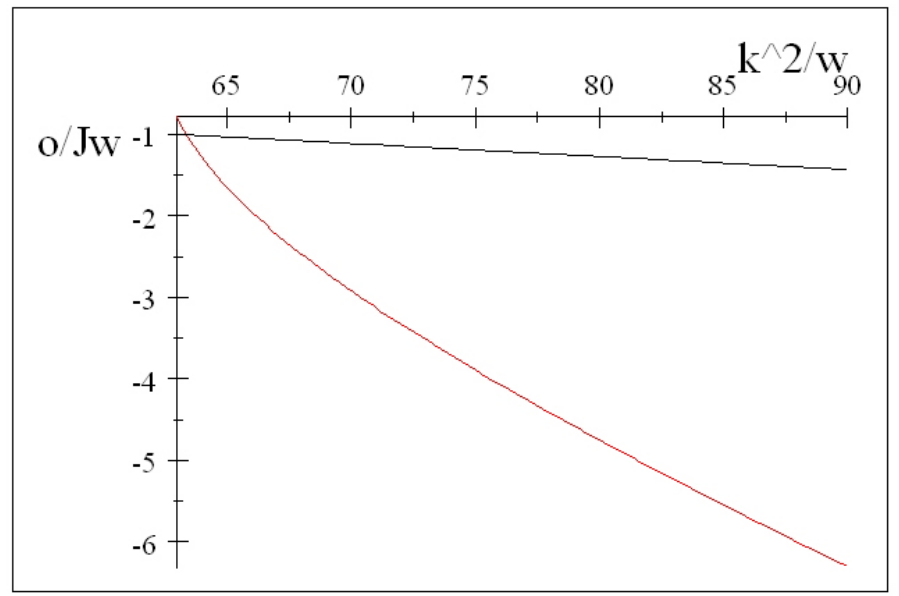

FIG. 2. The dependence of the real part of $o / K$ on $k^{2} / w$ (black) and its imaginary part (red) is presented at range of imaginary root expression for the same parameters values.

\section{Stationary solution}

\subsection{Homogeneous equation}

Stationary solutions for $\alpha \ll 1$ of the system:

$$
\begin{gathered}
Z_{t}=J \lambda W-K Z \alpha-J W_{z z}-J \alpha Z_{z z}+J Z \alpha \lambda, \\
W_{t}=J Z_{z z}+K Z-J Z \lambda-J \alpha W_{z z}+J \alpha \lambda W,
\end{gathered}
$$

is conventionally expressed in terms of the coordinate $\xi=z-c t$. We write: $Z_{t}=-c Z_{\xi}, Z_{z z}=Z_{\xi \xi}$, and get

$$
\begin{gathered}
-c Z_{\xi}=J \lambda W-K Z \alpha-J W_{\xi \xi}-J \alpha Z_{\xi \xi}+J Z \alpha \lambda, \\
-c W_{\xi}=J Z_{\xi \xi}+K Z-J Z \lambda-J \alpha W_{\xi \xi}+J \alpha \lambda W,
\end{gathered}
$$

which is the system of linear ODE. Its solution is searched by plugging the functions: $Z=A \exp (b \xi), W=$ $B \exp (b \xi)$ into the system, having:

$$
-c b A=J \lambda B-K A \alpha-J b^{2} B-J \alpha b^{2} A+J A \alpha \lambda,
$$


and:

$$
-c b B=J b^{2} A+K A-J \lambda A-J \alpha b^{2} B+J \alpha \lambda B .
$$

Solving the: equation (83) with respect to $B$, gives for $b^{2} \neq \lambda$ :

$$
B=\frac{1}{J \lambda-J b^{2}}\left(A K \alpha-A b c-A J \alpha \lambda+A J b^{2} \alpha\right) .
$$

Next, if, $-J \lambda+J a^{2} \neq 0$, plugging $B$ in (84) yields:

$$
\begin{aligned}
J^{2} b^{4} \alpha^{2}+J^{2} b^{4}-2 J^{2} b^{2} \alpha^{2} \lambda-2 J^{2} b^{2} \lambda+J^{2} \alpha^{2} \lambda^{2}+J^{2} \lambda^{2}-2 J b^{3} c \alpha+K J b^{2} \alpha^{2}+ \\
K J b^{2}+2 J b c \alpha \lambda-K J \alpha^{2} \lambda-K J \lambda+b^{2} c^{2}-K b c \alpha=0 .
\end{aligned}
$$

From this equation it follows the relation, that determine link between the velocity of the $k$-mode $c$ and $b$. Solving it with respect to $c$ we write:

$$
c=\frac{1}{2 b}\left(K \alpha-2 J \alpha \lambda+2 J b^{2} \alpha \pm \sqrt{-4 J^{2} \lambda^{2}+K^{2} \alpha^{2}-4 J^{2} b^{4}+8 J^{2} b^{2} \lambda+4 J K \lambda-4 J K b^{2}}\right) .
$$

Plugging $Z, W$ into the expression for $\Omega$, we write the transverse mode term as:

$$
Z+i W=A \exp (b \xi)\left[1+\frac{i}{J \lambda-J b^{2}}\left(K \alpha-b c-J \alpha \lambda+J b^{2} \alpha\right)\right] .
$$

We denote the roots of the under-square-root bi-quadratic expression as:

arriving at:

$$
\begin{aligned}
& \frac{1}{2}\left(\lambda-\sqrt{\lambda^{2}-2 J \lambda^{2}+J^{2} \lambda^{2}+K^{2}+\frac{K^{2} \alpha^{2}}{J}+2 K \lambda-2 J K \lambda}+J \lambda-K\right)=d_{1}, \\
& \frac{1}{2}\left(\lambda+\sqrt{\lambda^{2}-2 J \lambda^{2}+J^{2} \lambda^{2}+K^{2}+\frac{K^{2} \alpha^{2}}{J}+2 K \lambda-2 J K \lambda}+J \lambda-K\right)=d_{2},
\end{aligned}
$$

$$
c=-\frac{1}{2 b}\left(K \alpha-\sqrt{-4 J\left(b^{2}-d_{1}\right)\left(b^{2}-d_{2}\right)}-2 J \alpha \lambda+2 J b^{2} \alpha\right) .
$$

We see, that the rhs is real, if $b^{2}$ lies between the roots $d_{1,2}$. If the roots are both positive, the case takes place for a real $b$, otherwise $b$ may be as real for $b^{2}>0$, as imaginary, if $b^{2}<0$. For a convenience of plotting, we rearrange the formula for the velocity as:

$$
\frac{c}{b}=-\frac{d_{1}}{2 b^{2}}\left(\frac{K \alpha}{d_{1}}-\sqrt{4 J\left(\frac{b^{2}}{d_{1}}-1\right)\left(\frac{d_{2}}{d_{1}}-\frac{b^{2}}{d_{1}}\right)}-\frac{2 J \alpha \lambda}{d_{1}}+2 J \alpha \frac{b^{2}}{d_{1}}\right) .
$$

The plot of $\frac{c}{b}$ as the function of $\frac{b^{2}}{d_{1}} \in[0,1]$, or $b \in\left[0, \sqrt{d_{1}}\right]$, is presented at the Fig. 3 for the following parameters choice: $K=7.77 \times 10^{6}, J=1.5 \times 10^{-11}$ in SI units and $\alpha=0.016$, see, e.g. [11]; next, we take: $\lambda_{0,1}=\frac{(2.4)^{2}}{\rho_{0}^{2}}$.

For estimation we choose $\rho_{0}=100 \mathrm{~nm}=10^{-7} \mathrm{~m}$, hence we get $\lambda_{0,1} \approx 5.8 \times 10^{14}$. It results in $d_{1}=-8.2121 \times 10^{6}$, $d_{2}=5.8 \times 10^{14}$.

6.1.1. Inhomogeneous equation. The rhs of mode evolution equation (76) in the case of constant magnetic field may be written as a shorthand $\left(\begin{array}{c}\alpha \gamma \widetilde{a}_{m \nu}(k, t) \\ -\gamma \widetilde{a}_{m \nu}(k, t)\end{array}\right)=\left(\begin{array}{c}\alpha \gamma a \\ -\gamma a\end{array}\right)$, where $\widetilde{a}_{m \nu}$ is defined by (74). Its particular solution, determined by constants $Z=A, W=B$ satisfies the system:

$$
\begin{gathered}
J \lambda B-K A \alpha+J A \alpha \lambda+\alpha \gamma a=0, \\
K A-J A \lambda+J \alpha \lambda B-\gamma a=0 .
\end{gathered}
$$

From the first equation of (89), we have:

Plugging it to the second one yields:

$$
B=\frac{1}{J \alpha \lambda}(a \gamma-A K+A J \lambda) .
$$

$$
\frac{1}{\alpha}(a \gamma-A K+A J \lambda)-A K \alpha+a \alpha \gamma+A J \alpha \lambda=0,
$$




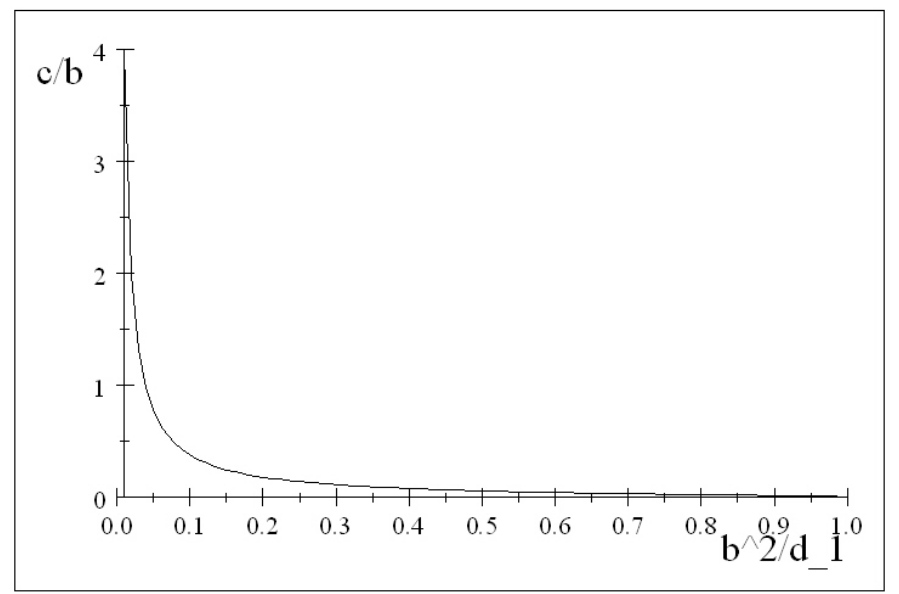

FIG. 3. The dependence of the DW velocity $c$ on $b^{2} / d_{1}$ within the range of real $c$, for $b>0$, for the chosen parameters values.

which gives:

$$
A=a \frac{\gamma}{K-J \lambda}, \quad B=0 .
$$

The general solution of an initial-boundary problem for (71) is, as was mentioned, the sum of general solution, see $(72,73)$, of homogeneous equation and the particular solution, defined by (89).

\subsection{Magnetization vector}

6.2.1. The magnetization vector components, general relations. In Cartesian coordinates the components of the magnetization vector are expressed in terms of complex field $\Omega$ as follows:

$$
\begin{gathered}
m^{z}=\frac{\exp (-\Re \Omega)-\exp (\Re \Omega)}{\exp (\Re \Omega)+\exp (-\Re \Omega)}, \\
m^{x}=\frac{\exp (\Omega)+\exp \left(\Omega^{*}\right)}{1+\exp [2 \Re \Omega]}, \\
m^{y}=-i \frac{\exp (\Omega)-\exp \left(\Omega^{*}\right)}{1+\exp [2 \Re \Omega]} .
\end{gathered}
$$

In cylindrical coordinates, the components appear as combinations of the Cartesian ones as:

$$
\begin{gathered}
m_{z}=m^{z}, \\
m_{\rho}=m^{x} \cos \phi+m^{y} \sin \phi \\
m_{\phi}=m^{x} \sin \phi+m^{y} \cos \phi
\end{gathered}
$$

for the geometry, see Fig. 4.

6.2.2. The domain wall. In approximations that were described through the presented text:

$$
m^{z} \simeq \frac{1-\exp \left(2 \Re \Omega^{\prime}\right)}{\exp \left(2 \Re \Omega^{\prime}\right)+1}=\frac{\exp (-R)-\exp (R)}{\exp (R)+\exp (-R)}=\tanh R,
$$

where:

$$
R(\varphi, \rho, z, t)=\sum_{\nu, m} \exp [i \nu \varphi] J_{\nu}\left(\epsilon_{m}(\nu) \frac{\rho}{\rho_{0}}\right) Z_{m \nu}^{l}(z, t)+c . c .
$$

The stationary solution motion is depicted at the Fig. 5, it shows the change of direction of magnetization to the opposite one at asymptotic "right" and "left" sides of a DW center $(\rho=0)$. 

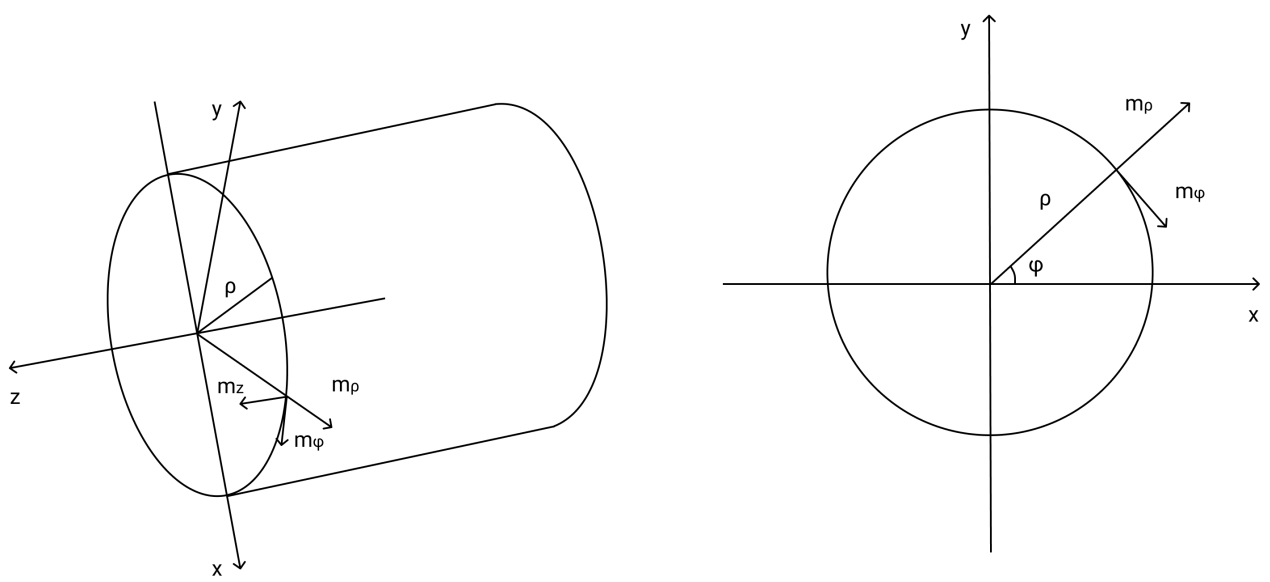

FIG. 4. Geometry. Vector $\vec{m}$ components in cylindrical coordinates, $\rho<\rho_{0}$

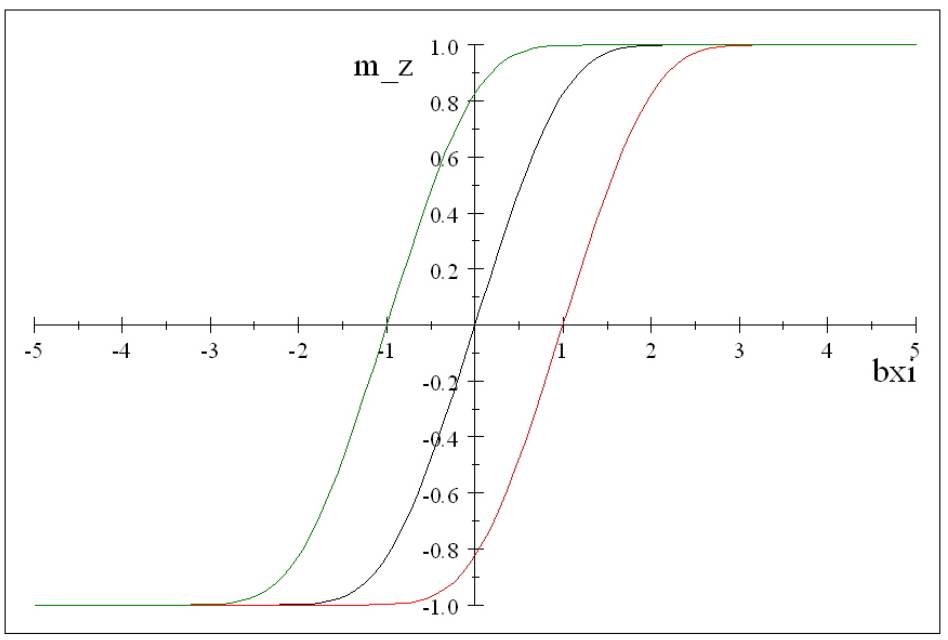

FIG. 5. The stationary wall motion plot at $b \xi=-1$ (green), 0 (black), 1 (red) for $m_{z}(\xi), \nu=0$, $n=1, \rho=0$

\section{Conclusion}

A detailed scheme for a DW creation dynamics, in terms of boundary-initial problems for zero and nonzero external magnetic fields, is proposed. The solutions of the problems are presented in the form of a combination of projections onto eigen subspaces of the evolution operators in $k$ - and $x$-domains in respect to Fourier transformation. The problem may be reformulated within the proposed scheme for anisotropy coefficient depending on $\rho$, $\phi$, as well as for coordinate-dependent external magnetic field.

\section{Acknowledgements}

The author thanks V. Rodionova for fruitful discussions.

\section{References}

[1] Ipatov M., Zhukova V., Zvezdin A.K., Zhukov A. Mechanisms of the ultrafast magnetization switching in bistable amorphous microwires, J. Appl. Phys., 2000, 106, 103902.

[2] Chizhik A.A., Zhukov A., Gonzalez A., Stupakiewicz A. Control of reversible magnetization switching by pulsed circular magnetic field in glass-coated amorphous microwires. Applied Physics Letters, 2018, 112 (7), 072407.

[3] Stupakiewicz A., Chizhik A., et al. Ultrafast Magnetization Dynamics in Metallic Amorphous Ribbons with a Giant Magnetoimpedance Response. Physical Review Applied, 2020, 13, 044058. 
[4] Badarneh M.H.A.G., Kwiatkowski J., Bessarab P.F. Mechanisms of energy efficient magnetization switching in a bistable nanowire. Nanosystems: Physics, Chemistry, Mathematics, 2020, 11 (3), P. 294-300.

[5] Rodioniova V., Zhukova M., et al. The defects influence on domain wall propagation in bistable glass-coated microwires. Physica B, 2012, 407, P. 1446-1449.

[6] Brown Jr. W.F. Micromagnetics, Domains, and Resonance. J. Appl. Phys., 1959, 106, 103902.

[7] Frenkel I., Dorfman J.N. Spontaneous and Induced Magnetisation in Ferromagnetic Bodies. Nature, 1930, 126, P. $274-278$.

[8] Landau L., Lifshitz E. On the theory of the dispersion of magnetic permeability in ferromagnetic bodies. Phys. Z. Sowjetunion, 1935, 8 (153), P. 101-114.

[9] Aharoni A. Introduction to the Theory of Ferromagnetism, Clarendon Press, Oxford, 1996, $192 \mathrm{p}$.

[10] Schryer N.L., Walker L.R. The motion of $180^{\circ}$ domain walls in uniform de magnetic fields. J. Appl. Phys., $1974,45,5406$.

[11] Vereshchagin M, Baraban I, Leble S., Rodionova V. Structure of head-to-head domain wall in cylindrical amorphous ferromagnetic microwire and a method of anisotropy coefficient estimation. Journal of Magnetism and Magnetic Materials, 2020, 504, 166646.

[12] Leble S. Waveguide Propagation of Nonlinear Waves. Impact of Inhomogeneity and Accompanying Effects, Springer, 2019, 288 p.

[13] Varga R., Zhukov A., et al. Fast magnetic domain wall in magnetic microwires. Phys. Rev. B, 2006, 74, 212405.

[14] Chizhik Al.,Zhukov A., Gonzalez J., and Stupakiewicz A. . Basic study of magnetic microwires for sensor applications: Variety of magnetic structures".Journal of Magnetism and Magnetic Materials, 2017. 422299 - 303,

[15] Omelyanchik A., Gurevich A., et al. Ferromagnetic glass-coated microwires for cell manipulation. Journal of Magnetism and Magnetic Materials, 2020, 242-245, P. 216-223.

[16] Amirov A., Baraban I., Panina L., Rodionova V. Direct Magnetoelectric Effect in a Sandwich Structure of PZT and Magnetostrictive Amorphous Microwires. Materials, 2020, 13 (4), 916.

[17] Vázquez M. Magnetic Nano- and Microwires: Design, Synthesis, Properties and Applications. Woodhead Publishing Series in Electronic and Optical Materials. Elsevier Science, 2015.

[18] Lakshmanan M. The fascinating world of the Landau-Lifshitz-Gilbert equation: an overview. Phil. Trans. R. Soc. A, 2011, 369, P. 1280-1300.

[19] Lakshmanan M., Nakamura K. Landau-Lifshitz Equation of Ferromagnetism: Exact Treatment of the Gilbert Damping. Phys. Rev. Lett., $1984, \mathbf{5 3}, 2497$

[20] Vereshchagin M. Structure of domain wall in cylindrical amorphous microwire. Physica B: Condensed Matter, 2018, 549, P. 91-93.

[21] Janutka A., Gawroński P. Structure of magnetic domain wall in cylindrical microwire. IEEE Transactions on Magnetics, 2015,51 (5), P. 1-6.

[22] Huening F., Backes A. Direct observation of large Barkhausen jump in thin Vicalloy wires. IEEE Magnetics Letters, $2020,11,2506504$.

[23] Leble S. Practical Electrodynamics with Advanced Applications, IOP Publishing, 2020.

[24] Baraban I., Leble S., Panina L., Rodionova V. Control of magneto-static and -dynamic properties by stress tuning in Fe-Si-B amorphous microwires with fixed dimensions. Journal of Magnetism and Magnetic Materials, 2019, 477, P. 415-419.

[25] Leble S.B., Rodionova V.V. Dynamics of Domain Walls in a Cylindrical Amorphous Ferromagnetic Microwire with Magnetic Inhomogeneities. Theor. Math. Phys., 2020, 202, P. 252-264.

[26] Kerimov M.K. Studies on the zeros of Bessel functions and methods for their computation. Comput. Math. and Math. Phys., 2014, 54, P. $1337-1388$

[27] Leble S., Perelomova A. Dynamical projectors method in hydro- and electrodynamics, CRC Press, Taylor and Francis group, 2018.

[28] Stano M., Fruchart O. Magnetic Nanowires and Nanotubes, Handbook of Magnetic Materials, 2018, DOI: 10.1016/bs.hmm.2018.08.002.

[29] Alam J., Bran C., et al. Cylindrical micro and nanowires: Fabrication, properties and applications. Journal of Magnetism and Magnetic Materials, 2020, 513, 167074.

[30] Popov I.Y. On the possibility of magnetoresistance governed by light. Nanosystems: physics, chemistry, mathematics, 2013, 4 (6), P. 795-799.

[31] Chizhik A., Gonzalez J., Zhukov A., Gawroński P. Study of length of domain walls in cylindrical magnetic microwires. Journal of Magnetism and Magnetic Materials, 2020, 512, 167060.

[32] Corte-León P., Gonzalez-Legarreta L., et al. Controlling the domain wall dynamics in Fe-, Ni- and Co-based magnetic microwires. Journal of Alloys and Compounds, 2020, 834, 155170. 